\title{
A Moore Bound for Simplicial Complexes
}

\author{
Alexander Lubotzky* Roy Meshulam ${ }^{\dagger}$
}

\begin{abstract}
Let $X$ be a $d$-dimensional simplicial complex with $N$ faces of dimension $(d-1)$. Suppose that any $(d-1)$-face of $X$ is contained in at least $k \geq d+2$ faces of $X$ of dimension $d$. Extending the classical Moore bound for graphs, it is shown that $X$ must contain a ball $B$ of radius at most $\left\lceil\log _{k-d} N\right\rceil$ such that $H_{d}(B ; \mathbb{R}) \neq 0$. The Ramanujan Complexes constructed by Lubotzky, Samuels and Vishne are used to show that this upper bound on the radius of $B$ cannot be improved by more then a multiplicative constant factor.
\end{abstract}

\section{Introduction}

Let $G=(V, E)$ be a graph on $n$ vertices. Let $\delta(G)$ denote the minimal degree in $G$ and let $g(G)=g$ denote the minimal length of a cycle in $G$. An easy counting argument (see e.g. Theorem IV.1 in [2]) shows that if $\delta(G)=k \geq 3$ then

$$
n \geq \begin{cases}1+\frac{k}{k-2}\left((k-1)^{\frac{g-1}{2}}-1\right) & g \text { odd } \\ \frac{2}{k-2}\left((k-1)^{\frac{g}{2}}-1\right) & g \text { even }\end{cases}
$$

Math Subject Classification. 52A35, 13F55.

Keywords and Phrases. Girth, Simplicial Homology, Ramanujan Graphs

${ }^{*}$ Institute of Mathematics, Hebrew University, Jerusalem 91904, Israel, and Institute for Advanced Study, Princeton, NJ 08540. e-mail: alexlub@math.huji.ac.il . Research supported by the Ambrose Monell Foundation and the Ellentuck Fund.

${ }^{\dagger}$ Department of Mathematics, Technion, Haifa 32000, Israel, and Institute for Advanced Study, Princeton, NJ 08540. e-mail: meshulam@math.technion.ac.il . Research supported by a State of New Jersey grant. 
This implies the classical Moore bound

Theorem A. $g(G)<2 \log _{k-1} n+2$.

Let $d_{G}(u, v)$ be the distance between the vertices $u$ and $v$ in the graph metric and let $B_{r}(v)=\left\{u \in V: d_{G}(u, v) \leq r\right\}$ denote the ball of radius $r$ around $v$. Define the acyclicity radius $r_{v}(G)$ of $G$ at the vertex $v$ to be the maximal $r$ such that the induced graph $G\left[B_{r}(v)\right]$ is acyclic. Let $r(G)=$ $\min _{v \in V} r_{v}(G)$, then $r(G)=\left\lfloor\frac{g(G)}{2}\right\rfloor-1$. The asymptotic version of Moore's bound is equivalent to the following

Theorem $\mathbf{A}_{1}$. If $\delta(G)=k \geq 3$ then for every $v \in V$

$$
r_{v}(G) \leq\left\lfloor\log _{k-1} n\right\rfloor .
$$

The best lower bound for the girth of $k$-regular graphs is given by the Ramanujan graphs of Lubotzky, Phillips and Sarnak [6]. For a fixed prime $p$, the construction in [6] provides a sequence of $(p+1)$-regular graphs $G_{i}=$ $\left(V_{i}, E_{i}\right)$ with $\left|V_{i}\right| \rightarrow \infty$ such that $g\left(G_{i}\right) \geq \frac{4}{3} \log _{p}\left|V_{i}\right|-O(1)$. A similar result was obtained by Morgenstern [9] for any prime power $q$. In terms of the acyclicity radius we therefore have:

Theorem $\mathbf{B}_{1}$. For every prime power $q$, there exists a sequence of $(q+1)$ regular graphs $G_{i}=\left(V_{i}, E_{i}\right)$ with $\left|V_{i}\right| \rightarrow \infty$ such that for every $v \in V$

$$
r_{v}\left(G_{i}\right) \geq \frac{2}{3} \log _{q}\left|V_{i}\right|-O(1)
$$

In this note we extend Theorems $\mathrm{A}_{1}$ and $\mathrm{B}_{1}$ to higher dimensional simplicial complexes. Let $X$ be a $d$-dimensional simplicial complex on the vertex set $V$. Let $H_{i}(X)$ denote the $i$-dimensional homology group of $X$ with some fixed field coefficients. For $0 \leq i \leq d$ let $X(i)=\{\sigma \in X: \operatorname{dim} \sigma=i\}$ and let $f_{i}(X)=|X(i)|$. For a subset of vertices $S \subset V$ let $X[S]$ denote the induced subcomplex on $S$. The degree of a $(d-1)$-simplex $\sigma \in X(d-1)$ is

$$
\operatorname{deg}(\sigma)=|\{\tau \in X(d): \sigma \subset \tau\}|
$$

Let $\delta(X)=\min \{\operatorname{deg}(\sigma): \sigma \in X(d-1)\}$. A complex $X$ is called $k$-regular if $\operatorname{deg}(\sigma)=\delta(X)=k$ for every $\sigma \in X(d-1)$. Denote by $B_{r}(v)$ the ball of radius $r$ around $v$ with respect to the graph metric on the 1-dimensional skeleton of 
$X$. Extending the notion of acyclicity radius to the higher dimensional setting we define $r_{v}(X)$ as the maximal $r$ such that $H_{d}\left(X\left[B_{r}(v)\right]\right)=0$, and $r(X)=$ $\min _{v \in V} r_{v}(X)$. The following result extends Theorem $\mathrm{A}_{1}$ to $d$-dimensional complexes.

Theorem $\mathbf{A}_{d}$. Let $X$ be a d-dimensional complex with $\delta(X)=k \geq d+2$. Then for any vertex $v \in V$ which is contained in some $(d-1)$-face

$$
r_{v}(X) \leq\left\lfloor\log _{k-d} f_{d-1}(X)\right\rfloor
$$

For the lower bound, we use the Ramanujan Complexes presented by Lubotzky, Samuels and Vishne in [8] to show:

Theorem $\mathbf{B}_{d}$. For $d \geq 1$ and $q$ a prime power, there exists a sequence of $d$-dimensional $(q+1)$-regular complexes $X_{i}$ on vertex sets $V_{i}$ with $\left|V_{i}\right| \rightarrow \infty$, such that for any $v \in V$

$$
r_{v}\left(X_{i}\right) \geq \frac{\log _{q}\left|V_{i}\right|}{2 d^{2}(d+2)}-1 .
$$

Theorem $\mathrm{A}_{d}$ is proved in Section 2, while Theorem $\mathrm{B}_{d}$ is established in Section 3. Note that Theorem $\mathrm{A}_{d}$ reduces to Theorem $\mathrm{A}_{1}$ when $d=1$. On the other hand, specializing theorem $\mathrm{B}_{d}$ for the case $d=1$, yields a somewhat weaker version of Theorem $B_{1}$ (The constant is $\frac{1}{6}$ rather then $\frac{2}{3}$ ). In Section 4 we discuss some open problems and suggestions for further research. One such challenge is to improve the constant in Theorem $\mathrm{B}_{d}$.

\section{The Upper Bound}

Proof of Theorem $\mathbf{A}_{d}$ : First note that if $Y$ is a $d$-dimensional complex such that $f_{d}(Y)>f_{d-1}(Y)$, then $H_{d}(Y) \neq 0$. Indeed, let $C_{i}(Y)$ denote the space of simplicial $i$-chains of $Y$. Then $\operatorname{dim} C_{d}(Y)=f_{d}(Y)>f_{d-1}(Y)=$ $\operatorname{dim} C_{d-1}(Y)$ implies that the boundary map $\partial: C_{d}(Y) \rightarrow C_{d-1}(Y)$ has a non-trivial kernel.

Let $v$ be a vertex which is contained in a $(d-1)$-simplex. Abbreviate $B_{t}=$ $B_{t}(v)$ and write $\alpha(t)=f_{d-1}\left(X\left[B_{t}\right]\right), \beta(t)=f_{d}\left(X\left[B_{t}\right]\right)$. Let

$$
\gamma(t)=\left|\left\{(\sigma, \tau): \sigma \in X\left[B_{t}\right](d-1), \tau \in X(d), \sigma \subset \tau\right\}\right| .
$$


Then

$$
\gamma(t)=\sum_{\sigma \in X\left[B_{t}\right](d-1)} \operatorname{deg}(\sigma) \geq f_{d-1}\left(X\left[B_{t}\right]\right) \cdot \delta(X) \geq \alpha(t) \cdot k
$$

For a $d$-simplex $\tau \in X(d)$ let $s(\tau)$ denote the number of $(d-1)$-simplices in $X\left[B_{t}\right]$ that are contained in $\tau$. Then

$$
s(\tau)= \begin{cases}d+1 & \tau \in X\left[B_{t}\right] \\ 0 & \tau \notin X\left[B_{t+1}\right]\end{cases}
$$

and $s(\tau) \leq 1$ if $\tau \in X\left[B_{t+1}\right]-X\left[B_{t}\right]$. Thus

$$
\begin{gathered}
\gamma(t)=\sum_{\tau \in X(d)} s(\tau) \leq(d+1) \beta(t)+(\beta(t+1)-\beta(t))= \\
d \beta(t)+\beta(t+1) .
\end{gathered}
$$

Let $m=r_{v}(X)$. Combining (3) and (4) we obtain that for all $t<m$

$$
k \alpha(t) \leq d \beta(t)+\beta(t+1) \leq d \alpha(t)+\alpha(t+1) .
$$

Hence

$$
\alpha(t+1) \geq(k-d) \alpha(t) \geq \cdots \geq(k-d)^{t} \alpha(1) .
$$

Since $v$ is contained in a $(d-1)$-face, it follows that $\alpha(1) \geq k d+1$. Thus

$$
(k d+1)(k-d)^{m-1} \leq \alpha(m) \leq f_{d-1}(X)
$$

and $m \leq\left\lfloor\log _{k-d} f_{d-1}(X)\right\rfloor$.

\section{The Lower Bound}

The proof of Theorem $\mathrm{B}_{d}$ depends on certain finite quotients of affine buildings constructed by Lubotzky, Samuels and Vishne [8], based on the Cartwright-Steger group [4] (see also [11] for a similar construction, as well as $[3,5,7]$ for related results). In Section 3.1 we recall the definition and some properties of affine buildings of type $\tilde{A}_{d-1}$. In Section 3.2 we describe the relevant finite quotients and show that they have a large acyclicity radius. 


\subsection{Affine Buildings of Type $\tilde{A}_{d-1}$}

Let $F$ be a local field with a valuation $\nu: F \rightarrow \mathbb{Z}$ and a uniformizer $\pi$. Let $\mathcal{O}$ denote the ring of integers of $F$ and $\mathcal{O} / \pi \mathcal{O}=\mathbb{F}_{q}$ be the residue field. A lattice $L$ in the vector space $V=F^{d}$ is a finitely generated $\mathcal{O}$-submodule of $V$ such that $L$ contains a basis of $V$. Two lattices $L_{1}$ and $L_{2}$ are equivalent if $L_{1}=\lambda L_{2}$ for some $0 \neq \lambda \in F$. Let $[L]$ denote the equivalence class of a lattice $L$. Two distinct equivalence classes $\left[L_{1}\right]$ and $\left[L_{2}\right]$ are adjacent if there exist representatives $L_{1}^{\prime} \in\left[L_{1}\right], L_{2}^{\prime} \in\left[L_{2}\right]$ such that $\pi L_{1}^{\prime} \subset L_{2}^{\prime} \subset L_{1}^{\prime}$. The affine building of type $\tilde{A}_{d-1}$ associated with $F$ is the simplicial complex $\mathcal{B}=\mathcal{B}_{d}(F)$ whose vertex set $\mathcal{B}^{0}$ is the set of equivalence classes of lattices in $V$, and whose simplices are the subsets $\left\{\left[L_{0}\right], \ldots,\left[L_{k}\right]\right\}$ such that all pairs $\left[L_{i}\right],\left[L_{j}\right]$ are adjacent. It can be shown that $\left\{\left[L_{0}\right], \ldots,\left[L_{k}\right]\right\}$ forms a simplex iff there exist representatives $L_{i}^{\prime} \in\left[L_{i}\right]$ such that

$$
\pi L_{k}^{\prime} \subset L_{0}^{\prime} \subset \cdots \subset L_{k}^{\prime}
$$

It is well known that $\mathcal{B}$ is a contractible $(d-1)$-dimensional simplicial complex and that the link of each vertex is isomorphic to the order complex $A_{d-1}\left(\mathbb{F}_{q}\right)$ of all non-trivial proper linear subspaces of $\mathbb{F}_{q}^{d}$ (see e.g. $[10,7]$ ). This implies that $\delta(\mathcal{B})=q+1$.

The type function $\tau: \mathcal{B}^{0} \rightarrow \mathbb{Z}_{d}$ is defined as follows. Let $\mathcal{O}^{d}$ be the standard lattice in $V$. For any lattice $L$, there exists $g \in \mathrm{GL}(V)$ such that $L=$ $g \mathcal{O}^{d}$. Define $\tau([L])=\nu(\operatorname{det}(g))(\bmod d)$. Let $\operatorname{dist}\left([L],\left[L^{\prime}\right]\right)$ denote the graph distance between $[L],\left[L^{\prime}\right] \in \mathcal{B}^{0}$ in the 1 -skeleton of $\mathcal{B}$. Let $\operatorname{dist}_{1}\left([L],\left[L^{\prime}\right]\right)$ denote the minimal $t$ for which there exist $[L]=\left[L_{0}\right], \ldots,\left[L_{t}\right]=\left[L^{\prime}\right]$ such that $\left[L_{i}\right]$ and $\left[L_{i+1}\right]$ are adjacent in $\mathcal{B}$ and $\tau\left(\left[L_{i+1}\right]\right)-\tau\left(\left[L_{i}\right]\right)=1$ for all $0 \leq i \leq t-1$.

Claim 3.1. For two lattices $L_{1}, L_{2}$

$$
\operatorname{dist}_{1}\left(\left[L_{1}\right],\left[L_{2}\right]\right) \leq(d-1) \operatorname{dist}\left(\left[L_{1}\right],\left[L_{2}\right]\right) \quad .
$$

Proof: This follows directly from (5). Alternatively, let $v_{1}, \ldots, v_{d}$ be a basis of $V$ and let $a_{1}, \ldots, a_{d}$ be integers such that $L_{1}=\oplus_{i=1}^{d} \mathcal{O} v_{i}$ and $L_{2}=$ $\oplus_{i=1}^{d} \pi^{a_{i}} \mathcal{O} v_{i}$. Then

$$
\operatorname{dist}\left(\left[L_{1}\right],\left[L_{2}\right]\right)=\max _{i} a_{i}-\min _{i} a_{i}
$$


and

$$
\operatorname{dist}_{1}\left(\left[L_{1}\right],\left[L_{2}\right]\right)=\sum_{i=1}^{d} a_{i}-d \min _{i} a_{i} .
$$

Now (6) follows from (7) and (8).

\subsection{Finite Quotients of Affine Buildings}

Let $q$ be a prime power and let $F$ be the local field $\mathbb{F}_{q}((y))$ with local ring $\mathcal{O}=\mathbb{F}_{q}[[y]]$. The construction of finite quotients of $\mathcal{B}=\mathcal{B}_{d}(F)$ in [8], depends on the remarkable Cartwright-Steger group $\Gamma<\mathrm{PGL}_{d}(F)$ (see [4]). We briefly recall the construction of $\Gamma$ and some of its properties.

Let $\phi: \mathbb{F}_{q^{d}} \rightarrow \mathbb{F}_{q^{d}}$ denote the Frobenius automorphism. Extend $\phi$ to $\mathbb{F}_{q^{d}}(y)$ by defining $\phi(y)=y$. Then $\phi$ is a generator of the cyclic Galois group $\operatorname{Gal}\left(\mathbb{F}_{q^{d}}(y) / \mathbb{F}_{q}(y)\right)$. Let $\mathcal{D}$ be the $d^{2}$-dimensional $\mathbb{F}_{q}(y)$-algebra given by $\mathcal{D}=\mathbb{F}_{q^{d}}[\sigma]$ with the relations $\sigma a=\phi(a) \sigma$ for all $a \in \mathbb{F}_{q^{d}}(y)$, and $\sigma^{d}=1+y$. $\mathcal{D}$ is a division algebra that splits over the extension field $F=\mathbb{F}_{q}((y))$. Denote $\mathcal{D}(F)=\mathcal{D} \otimes F$, then there is an isomorphism $\mathcal{D}(F) \cong M_{d}(F)$ which in turn induces an isomorphism

$$
\mathcal{D}(F)^{\times} / Z\left(\mathcal{D}(F)^{\times}\right) \cong \mathrm{PGL}_{d}(F)
$$

Let $b_{1}=1-\sigma^{-1} \in \mathcal{D}^{\times}$, and for $u \in \mathbb{F}_{q^{d}}^{*}$ let $b_{u}=u^{-1} b_{1} u$. Let $g_{u} \in$ $\mathcal{D}(F)^{\times} / Z\left(\mathcal{D}(F)^{\times}\right)$denote the image of $b_{u}$ under the quotient map. The Cartwright-Steger group $\Gamma$ is the subgroup of $\mathcal{D}(F)^{\times} / Z\left(\mathcal{D}(F)^{\times}\right)$generated by $\left\{g_{u}: u \in \mathbb{F}_{q^{d}}^{*}\right\}$. Utilizing the isomorphism (9), we also regard $\Gamma$ as a subgroup of $\mathrm{PGL}_{d}(F)$. We shall use the following properties of $\Gamma$.

Theorem 3.2. (Cartwright and Steger [4])

a) $\Gamma$ acts simply transitively on the vertices of $\mathcal{B}$.

b) Let $L_{0}=\mathcal{O}^{d}$. Then for $g \in \Gamma$

$$
\operatorname{dist}_{1}\left(g\left[L_{0}\right],\left[L_{0}\right]\right)=\min \left\{t: g=g_{u_{1}} \cdots g_{u_{t}} \text { for some } u_{1}, \ldots, u_{t} \in \mathbb{F}_{q^{d}}^{*}\right\}
$$


The action of $\mathcal{D}$ upon itself by conjugation gives rise to a representation

$$
\rho: \mathcal{D}(F)^{\times} \rightarrow \mathrm{GL}_{d^{2}}(F)
$$

which factors through $\mathcal{D}(F)^{\times} / Z\left(\mathcal{D}(F)^{\times}\right)$. Let $\xi_{0}, \ldots, \xi_{d-1}$ be a normal basis of $\mathbb{F}_{q^{d}}$ over $\mathbb{F}_{q}$, then $\left\{\xi_{i} \sigma^{j}\right\}_{i, j=0}^{d-1}$ is a basis of $\mathcal{D}(F)$ over $F$. An explicit computation (see Eq. (9) on page 975 in [8]) shows that with respect to this basis, $\rho\left(b_{u}\right)$ is a $d^{2} \times d^{2}$ matrix whose entries are linear polynomials in $\frac{1}{y}$ over $\mathbb{F}_{q}$. Let $h(\lambda) \in \mathbb{F}_{q}[\lambda]$ be an irreducible polynomial which is prime to $\lambda(1+\lambda)$, and let $f=h\left(\frac{1}{y}\right) \in R_{0}=\mathbb{F}_{q}\left[\frac{1}{y}\right]$ and $I=f R_{0}$. Write $\mathbf{1}_{d^{2}}$ for the $d^{2} \times d^{2}$ identity matrix. Let

$$
\Gamma(I)=\left\{\gamma \in \Gamma: \rho(\gamma) \equiv \mathbf{1}_{d^{2}}(\bmod f)\right\} .
$$

This subgroup coincides with the congruence subgroup $\Gamma(I)$ as defined in Eq. (15) on p.979 in [8]. In particular $\Gamma / \Gamma(I)$ is isomorphic to a subgroup of $\mathrm{PGL}_{d}\left(R_{0} / f R_{0}\right)$. Let $\mathcal{B}_{I}=\Gamma(I) \backslash \mathcal{B}$ denote the resulting quotient complex. The vertex set $\mathcal{B}_{I}^{0}$ of $\mathcal{B}_{I}$ is the set of orbits of $\mathcal{B}^{0}$ under $\Gamma(I)$, i.e.

$$
\mathcal{B}_{I}^{0}=\left\{\Gamma(I)[L]:[L] \in \mathcal{B}^{0}\right\} .
$$

A subset $\left\{\Gamma(I)\left[L_{0}\right], \ldots, \Gamma(I)\left[L_{k}\right]\right\}$ forms a simplex in $\mathcal{B}_{I}$ iff there exist $g_{0}, \ldots, g_{k} \in$ $\Gamma(I)$ such that $\left\{g_{0}\left[L_{0}\right], \ldots, g_{k}\left[L_{k}\right]\right\}$ is a simplex in $\mathcal{B}$.

Note that

$$
\left|\mathcal{B}_{I}^{0}\right|=(\Gamma: \Gamma(I)) \leq\left|\mathrm{PGL}_{d}\left(R_{0} / f R_{0}\right)\right| .
$$

Let $L$ be a lattice, and let

$$
\ell_{I}=\min \{\operatorname{dist}([L], g[L]): 1 \neq g \in \Gamma(I)\} .
$$

Clearly $\ell_{I}$ is independent of $L$ since $\Gamma$ is transitive and $\Gamma(I) \triangleleft \Gamma$.

\section{Proposition 3.3.}

$$
\ell_{I} \geq \frac{\log _{q}\left|\mathcal{B}_{I}^{0}\right|}{(d-1)\left(d^{2}-1\right)}
$$

Proof: Let $t=\operatorname{dist}_{1}\left(g\left[L_{0}\right],\left[L_{0}\right]\right)$. By Theorem 3.2b) there exist $u_{1}, \ldots, u_{t} \in$ $\mathbb{F}_{q^{d}}^{*}$ such that $g=g_{u_{1}} \cdots g_{u_{t}}$. Let $C=\left(c_{i j}\right)=\rho\left(b_{u_{1}}\right) \cdots \rho\left(b_{u_{t}}\right)$. The $c_{i j}$ 's are polynomials in $\mathbb{F}_{q}\left[\frac{1}{y}\right]$ of degree at most $t$ in $\frac{1}{y}$. By assumption $g \in \Gamma(I)$, 
hence $C=\mathbf{1}_{d^{2}}+f E$ for some $E \in M_{d^{2}}\left(R_{0}\right)$. If $c_{i j} \neq 0$ for some $i \neq j$, then $t \geq \operatorname{deg}_{1 / y}\left(c_{i j}\right) \geq \operatorname{deg}_{1 / y}(f)$. Otherwise $C$ is a diagonal matrix. If it is a scalar matrix, then it must be the identity as $\Gamma$, being a lattice in $\operatorname{PGL}_{d}(F)$, has trivial center. Thus we can assume $C$ is diagonal and non-scalar. Choose $i, j$ such that $c_{i i} \neq c_{j j}$, then $t \geq \operatorname{deg}_{1 / y}\left(c_{i i}-c_{j j}\right) \geq \operatorname{deg}_{1 / y}(f)$. Thus, by (6)

$$
\begin{gathered}
\operatorname{dist}\left(\left[L_{0}\right], g\left[L_{0}\right]\right) \geq \frac{1}{d-1} \operatorname{dist}_{1}(g[L],[L]) \geq \\
\frac{\operatorname{deg}_{1 / y}(f)}{(d-1)} \geq \frac{\log _{q}\left|\mathrm{PGL}_{d}\left(R_{0} / f R_{0}\right)\right|}{(d-1)\left(d^{2}-1\right)} \geq \\
\frac{\log _{q}\left|\mathcal{B}_{I}^{0}\right|}{(d-1)\left(d^{2}-1\right)} .
\end{gathered}
$$

Proof of Theorem $\mathbf{B}_{d-1}$ : Choose a sequence of irreducible polynomials $h_{i}(\lambda) \in \mathbb{F}_{q}[\lambda]$ such that $\left(h_{i}, \lambda(1+\lambda)=1\right.$ and $\operatorname{deg} h_{i} \rightarrow \infty$. Let $I_{i}=h_{i}\left(\frac{1}{y}\right) R_{0}$ and let $X_{i}=\mathcal{B}_{I_{i}}$. The quotient map $\mathcal{B} \rightarrow X_{i}$ is clearly an isomorphism on balls of radius at most $\frac{\ell_{I_{i}}}{2}-1$ in $\mathcal{B}$. Since $\mathcal{B}$ is contractible, it follows from Proposition 3.3 that for any vertex $v \in X_{i}^{0}$

$$
\begin{gathered}
r_{v}\left(X_{i}\right) \geq \frac{\ell_{I_{i}}}{2}-1 \geq \\
\frac{\log _{q}\left|X_{i}^{0}\right|}{2(d-1)\left(d^{2}-1\right)}-1 .
\end{gathered}
$$

We complete the proof by noting that if $i$ is sufficiently large then $\ell_{I_{i}} \geq 4$, hence $X_{i}$ is $(d-1)$-dimensional and $\delta\left(X_{i}\right)=\delta(\mathcal{B})=q+1$.

\section{Concluding Remarks}

We proved a higher dimensional extension of the Moore bound, and showed that the Ramanujan Complexes constructed in [8] imply that this bound is tight up to a multiplicative factor. We mention several problems that arise from these results. 
1. In Section 3.2 it is shown that for appropriately chosen ideals $I_{i} \triangleleft \mathbb{F}_{q}\left[\frac{1}{y}\right]$, the $(d-1)$-dimensional quotient complexes $X_{i}=\mathcal{B}_{I_{i}}$ satisfy

$$
r_{v}\left(X_{i}\right) \geq C(d-1) \log _{q}\left|X_{i}^{0}\right|-1
$$

with $C(d-1)=\frac{1}{2(d-1)\left(d^{2}-1\right)}$. It seems likely that a more careful choice of the $I_{i}$ 's will lead to an improved bound on the constant. (Recall that in the 1-dimensional case, Ramanujan graphs [6] give the constant $\frac{2}{3}$, while $\left.C(1)=\frac{1}{6}\right)$.

2. While the construction of Ramanujan Graphs and the proof of Theorem $\mathrm{B}_{1}$ depend on number theoretic tools, there is an elementary (but nonconstructive) argument due to Erdős and Sachs (see e.g. Theorem III.1.4 in [1]) that shows the existence of a sequence of $k$-regular graphs $G_{i}=\left(V_{i}, E_{i}\right)$ with $\left|V_{i}\right| \rightarrow \infty$ such that $r\left(G_{i}\right) \geq \frac{1}{2} \log _{k-1}\left|V_{i}\right|-O(1)$. It would be interesting to obtain a similar result in the higher dimensional setting.

\section{References}

[1] B. Bollobás, Extremal Graph Theory, Academic Press, London, 1978.

[2] B. Bollobás, Modern Graph Theory, Graduate Texts in Mathematics, Springer Verlag, New York, 1998.

[3] D. Cartwright, P. Solé, A. Żuk, Ramanujan geometries of type $A_{n}$. Discrete Math. 269 (2003), 35-43.

[4] D. Cartwright and T. Steger, A family of $\tilde{A}_{n}$-groups, Israel J. Math. 103(1998), 125-140.

[5] W.-C. W. Li, Ramanujan hypergraphs, Geom. Funct. Anal. 14 (2004), 380-399.

[6] A. Lubotzky, R. Phillips, P. Sarnak, Ramanujan Graphs, Combinatorica 8(1988) 261-277.

[7] A. Lubotzky, B. Samuels, U. Vishne, Ramanujan complexes of type $\mathrm{A}_{d}$, Israel J. Math, to appear. 
[8] A. Lubotzky, B. Samuels, U. Vishne, Explicit constructions of Ramanujan complexes of type $\mathrm{A}_{d}$, European J. Combin. 26 (2005) 965-993.

[9] M. Morgenstern, Existence and explicit constructions of $q+1$ regular Ramanujan graphs for every prime power $q$, J. Combin. Theory Ser. B 62 (1994) 44-62.

[10] M. Ronan, Lectures on Buildings, Perspectives in Mathematics Vol. 7, Academic Press, London 1989.

[11] A. Sarveniazi, Ramanujan $\left(n_{1}, n_{2}, \ldots, n_{d-1}\right)$-regular hypergraphs based on Bruhat-Tits buildings of type $\tilde{A}_{d-1}$, arxiv.org/math.NT/0401181. 